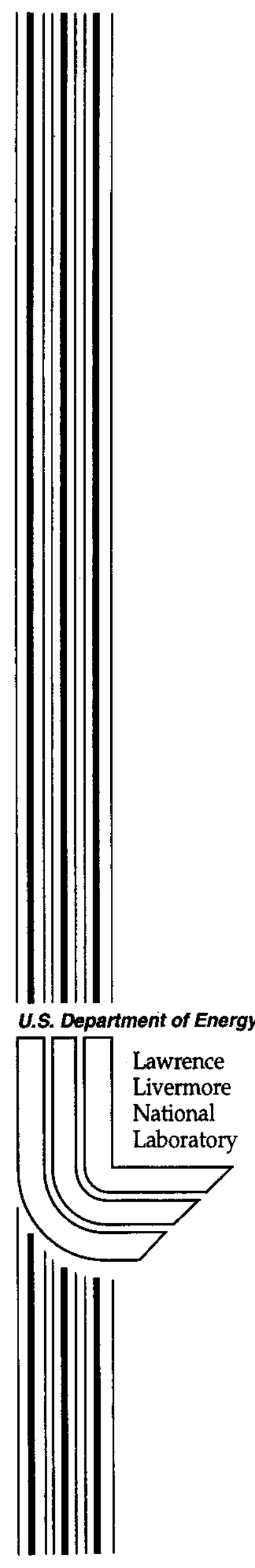

Preprint

UCRL-JC-139170

\title{
Effect of Magnetic Geometry on ELM Heat Flux Profiles
}

C.J. Lasnier, A.W. Leonard, T.W. Petrie, J.G. Watkins

This article was sybmitted to $14^{\text {th }}$ International Plasma Surface Interactions Conference, Rosenheim, Germany, May 22-26, 2000

\section{May 15, 2000}




\section{DISCLAIMER}

This document was prepared as an account of work sponsored by an agency of the United States Government. Neither the United States Government nor the University of California nor any of their employees, makes any warranty, express or implied, or assumes any legal liability or responsibility for the accuracy, completeness, or usefulness of any information, apparatus, product, or process disclosed, or represents that its use would not infringe privately owned rights. Reference herein to any specific commercial product, process, or service by trade name, trademark, manufacturer, or otherwise, does not necessarily constitute or imply its endorsement, recommendation, or favoring by the United States Government or the University of California. The views and opinions of authors expressed herein do not necessarily state or reflect those of the United States Government or the University of California, and shall not be used for advertising or product endorsement purposes.

This is a preprint of a paper intended for publication in a journal or proceedings. Since changes may be made before publication, this preprint is made available with the understanding that it will not be cited or reproduced without the permission of the author.

This report has been reproduced directly from the best available copy.

Available to DOE and DOE contractors from the Office of Scientific and Technical Information

P.O. Box 62, Oak Ridge, TN 37831

Prices available from (423) 576-8401 http://apollo.osti.gov/bridge/

Available to the public from the National Technical Information Service

U.S. Department of Commerce 5285 Port Royal Rd., Springfield, VA 22161 http://www.ntis.gov/

\section{OR}

Lawrence Livermore National Laboratory Technical Information Department's Digital Library http://www.llnl.gov/tid/Library.html 


\title{
Effect of magnetic geometry on ELM heat flux profiles
}

\author{
C.J. Lasnier, ${ }^{a}$ A.W. Leonard, ${ }^{b}$ T.W. Petrie, ${ }^{b}$ and J.G. Watkins ${ }^{c}$ \\ ${ }^{a}$ Lawrence Livermore National Laboratory, Livermore, California 94551 \\ ${ }^{b}$ General Atomics, P.O. Box 85608, San Diego, California 92186-5608 \\ cSandia National Laboratories, Albuquerque, New Mexico
}

\begin{abstract}
In this paper we explore how precisely the magnetic up/down symmetry must be controlled to insure sharing of edge localized mode (ELM) heat flux between upper and lower divertors in a double-null tokamak. We show for DIII-D, using infrared thermography, that the spatial distribution of Type-I ELM energy is less strongly affected by variations in magnetic geometry than is the time-averaged peak heat flux in attached discharges. The degree of control necessary to share ELM heat flux deposition equally between divertors was less stringent than the control needed to balance the time averaged heat flux. ELM energy is transported more than four times further into the scrape-off layer than the time-averaged heat flux.
\end{abstract}

Keywords: ELM, Heat flux, Divertor, Thermography

\section{Introduction}

In future high-power tokamaks a prominent design consideration is coping with ELM heat flux on the divertor plate. If the ELM energy flux exceeds $1.5 \mathrm{MJ} / \mathrm{m}^{2}$, ablation of the divertor plate will be unacceptably high [1]. One strategy is to make a double-null tokamak and attempt to share the ELM heat flux between the upper and lower divertors. However, the sharing of the ELM heat flux between the upper and lower divertors is strongly affected by the magnetic balance between the divertors. 
In DIII-D, the up-down magnetic balance is expressed by the quantity $\operatorname{dr}_{S E P}$, which is the radial distance at the outer midplane between the two flux surfaces connected to the two nulls. A large negative value of $\mathrm{dr} S E P$ represents a lower single null, a large positive value represents an upper single null, and $\operatorname{dr}_{S E P}=0$ represents a magnetically balanced double-null.

The benefits of sharing heat flux between two divertors apply to the time averaged heat flux as well as the ELM heat flux. The magnetic control required to achieve heat flux sharing is a critical part of the design for a high-power tokamak with double-null divertor configuration, or with a secondary null which could direct significant heat flux to the nearby wall [2]. We investigated whether the sharing of ELM heat flux required the same degree of magnetic control. This is of particular concern in the ITER device in which the planned poloidal field coils are outside the toroidal field coils.

\section{Experimental method}

We changed the up-down magnetic balance $\mathrm{dr}_{S E P}$ between upper and lower divertors and observed the effect on the distribution of ELM heat flux on divertor surfaces. The value of $\operatorname{dr}_{S E P}$ was varied from one discharge to the next, or within a single discharge. We used ELMing $\mathrm{H}$-mode discharges with plasma current of $1.4 \mathrm{MA}$, toroidal field of $2.0 \mathrm{~T}$, ion $\nabla \mathbf{B}$ drift downward, and core density approximately $5 \times 10^{13} \mathrm{~m}^{-3}$.

Heat flux was measured using infrared thermography with two cameras, one viewing the upper divertor and the other viewing the lower divertor. The heat flux profiles on the surface were calculated from the surface temperature data using a method similar to that discussed in previous publications $[3,4]$. From the heat flux profiles we calculated the energy deposited on the surface by integrating the profiles over the time of the ELM.

The camera views and divertor geometry are shown in Fig. 1. The cameras are actually at two different toroidal locations, but are shown on the same cross-section for simplicity. Each camera gives surface temperature profiles with a time resolution of $125 \mu \mathrm{s}$. 


\section{Analysis}

We used the energy deposited by an ELM to calculate the energy balance ratio between the upper and lower divertors as $R_{E}=\left(E_{u p}-E_{l o w}\right) /\left(E_{u p}+E_{l o w}\right)$. Here $E_{u p}$ and $E_{l o w}$ are the energies deposited on the upper and lower divertor plates respectively by an ELM. This value approaches unity for an upper single-null and -1 for a lower single-null. The ELM energies are obtained by integrating the surface heat flux over the radial profile and over the duration of the ELM, and assuming toroidal symmetry.

In Fig. 2 we plot $R_{E}$ versus dr ${ }_{S E P}$ for ELMs, along with the ratio of time averaged peak heat fluxes calculated in a similar way: $R_{q}=\left(q_{u p}-q_{l o w}\right) /\left(q_{u p}+q_{l o w}\right)$ for attached and detached discharges. We use $\mathrm{q}_{\mathrm{up}}$ and $\mathrm{q}_{\text {low }}$ to denote the time-averaged heat flux at the peak of the profile on the upper and lower divertor plates respectively. The energy balance ratio $R_{E}$ is plotted on the left axis and the peak heat flux ratio $R_{\mathrm{q}}$ is plotted on the right axis. The curves are hyperbolic tangent fits to the data.

Notice that the ELM energy ratio varies much more slowly with drSEP than the attached peak heat flux ratio, but is similar to the dependence of detached peak heat flux on $\mathrm{dr}_{S E P}$. The scale length of the transition from downward heat flux to upward heat flux for the attached discharges $(0.4 \mathrm{~cm})$ is similar to the scale length for heat flux penetration into the scrape off layer of $0.5-0.6 \mathrm{~cm}$ [5]. The drSEP scale length to change the ELM energy flux deposition is much larger at $1.9 \mathrm{~cm}$. For comparison, the $\mathrm{dr}_{S E P}$ scale length for the detached time averaged peak heat flux is $2.2 \mathrm{~cm}[6]$.

Two factors are most important in preventing $R_{E}$ from reaching -1 or +1 . First, the ELM energy deposition still shows some energy in the upper divertor when drSEP is biased downward. This is primarily because of heat deposited on the edge of the upper pump baffle. The flux surface that is $4 \mathrm{~cm}$ from the separatrix when mapped to the midplane did not clear this baffle completely even for large negative values of drSEP. Secondly, The shape of the curve is also 
affected by the fact that not all of the inner strike point heat flux in the lower divertor was within the field of view of the camera. We estimate that this contributes a few percent error to $R_{E}$.

Figure 3 shows heat flux profiles as a function of time for a single ELM in the lower and upper divertor. Also shown are the corresponding geometries of the tile surfaces in the divertors. The positions where the separatrix intersects the plate are shown as dotted lines. For this discharge drSEP $=-2.2 \mathrm{~cm}$ and $\mathrm{R}_{\mathrm{q}}=-0.35$. Even though this is a downward magnetic bias we see residual heat on the edge of the upper baffle. We also see the inner lower strike point profile cut off by the edge of the field of view of the camera. Most of the heat is deposited in the lower divertor for this large negative $\mathrm{dr}$ SEP.

The overall heat flux profile in the lower divertor is quite broad. However, we see from the profiles that the peak ELM heat flux does not appear in the private flux region. The observation is consistent with what we expect from the magnetic geometry, with heat primarily flowing along field lines. The conformity of the deposition profiles to the expected positions is evidence that the magnetic flux surfaces are not moving drästically during ELMs in DIII-D.

Figure 4 shows similar heat flux profiles for an ELM during an upward biased discharge. The contrast and brightness have been expanded for the lower divertor image. We see very little heat flux in the lower divertor. In the upper divertor the heat flux is again localized near the edge of the outer baffle at major radius $\mathrm{R}=138 \mathrm{~cm}$.

In Figs. 5 and 6 we show energy flux profiles in the lower divertor and upper divertor respectively for values of $\mathrm{dr}_{S E P}$ ranging from $-3.8 \mathrm{~cm}$ to $+3.4 \mathrm{~cm}$. For the largest positive values of $\operatorname{dr} S E P$ the energy flux profile in the lower divertor near the separatrix nearly vanishes. There is some residual energy flux far from the separatrix. We also see in these profiles where the inner lower energy flux profile was cut off by the edge of the field of view of the camera.

In the upper divertor profiles we see that for large negative values of $\mathrm{dr} S E P$ the peak energy flux is greatly reduced at both the inner and outer strike points. For large positive values of $\mathrm{dr}_{S E P}$, the heating is peaked near the pump baffle, rather than the broad profile seen in the lower 
divertor. The reason for this difference is not yet completely understood, but seems to depend on the presence of the baffle.

In both the upper and lower divertor the heating near the separatrix is most strongly affected by the change in $\mathrm{dr}_{S E P}$. This is because the flux surfaces near the separatrix change relative position at the midplane when $\operatorname{dr}_{S E P}$ is changed. As the heat flux diffuses outward from the main plasma, heat will be directed primarily along the first flux surfaces encountered which connect with a divertor. The flux surfaces farther out which are connected to the other divertor receive less of the heat and so conduct less heat to the corresponding divertor. 


\section{Conclusions}

We find that the ELM heat penetrates more than four times farther into the scrape off layer than the time averaged heat flux. A correspondingly greater change in $\operatorname{dr} S E P$ is necessary to direct the ELM heat flux to the opposite divertor. This means a less precise control system can still exert adequate control over the ELM heat flux balance, compared to the precision required for balancing the time averaged heat flux between two divertors. This bodes well for those future double-null tokamaks that cannot exert fine control over $\mathrm{dr} S E P$.

\section{Acknowledgments}

Work supported by the U.S. Department of Energy at Lawrence Livermore National Laboratory under Contract Nos. DE-AC03-99ER54463, W-7405-ENG-48, and DE-AC0494AL85000.

\section{References}

[1] H.D. Pacher, "E9. Disruption and ELM Erosion," Appendix E9, Section 1.7 (Divertor), ITER Design Description Document, ITER No. "G 17 DDD 1 96-08-21 W2.1," August 1996.

[2] G. Janeshitz, "Physics Issues (H-mode Pedestal, ITBs) for RC-ITER," Memo G 17 MD 117 98-07-16 W 0.2, RC-ITER, 1998.

[3] D.N. Hill, R. Ellis, W. Ferguson, et al., Rev. Sci. Instrum. 59, (1988) 1878-1880.

[4] C.J. Lasnier, D.N. Hill, T.W. Petrie, et al., Nucl. Fusion 38, (1998) 1225-1249.

[5] T.W. Petrie, M.E. Fenstermacher, S.L. Allen, T.N. Carlstrom, R.J. Groebner, C.J. Lasnier, A.W. Leonard, M.A. Mahdavi, R. Maingi, R. Moyer, T.L. Rhodes, D.M. Thomas, J.W. 
Watkins, W.P. West, and the DIII-D Team, 26th EPS conference on Controlled Fusion and Plasma Physics European Physical Society, Maastricht, the Netherlands, (1999), 23J, 1237.

[6] T.W. Petrie, et al., "The Effect of Divertor Magnetic Balance on H-mode Performance in DIII-D," these proceedings. 


\section{Figure Captions}

Fig. 1. Infrared camera views and divertor geometry. The dashed lines show the view of the lower divertor, and the dotted lines depict the view of the upper divertor. The cameras are actually located at different toroidal positions.

Fig. 2. a) ELM Energy balance ratio versus $\mathrm{dr}_{S E P}$ and b) heat flux balance ratio versus $\mathrm{dr}_{S E P}$ for peak attached heat flux (time-averaged), and peak detached heat flux (time-averaged). The curves are hyperbolic tangent fits. The qualitative behavior of the ELM energy with varying $\operatorname{dr}_{S E P}$ is similar to the time-averaged attached peak heat flux, but the scale length to change from one divertor to the other is much greater, and similar to the detached time-averaged peak heat flux.

Fig. 3. Heat flux profiles versus time for a single ELM in the lower and upper divertor. The corresponding tile structures in the divertors are also shown. This discharge was magnetically biased downward.

Fig. 4. Heat flux profiles versus time for an upward biased discharge. The divertor structures are also shown. The brightness and contrast were expanded for the lower divertor image.

Fig. 5. Lower divertor energy flux profiles for various values of $\operatorname{dr}$ SEP. The separatrix locations are shown. For the largest positive drSEP the energy flux near the separatrix nearly vanishes.

Fig. 6. Upper divertor energy flux profiles for various values of drSEP. The locations of the separatrix and the edge of the upper pump baffle are shown. For large $\operatorname{dr}_{S E P}$, the heating is peaked near the pump baffle, rather than the broad profile seen in the lower divertor. 


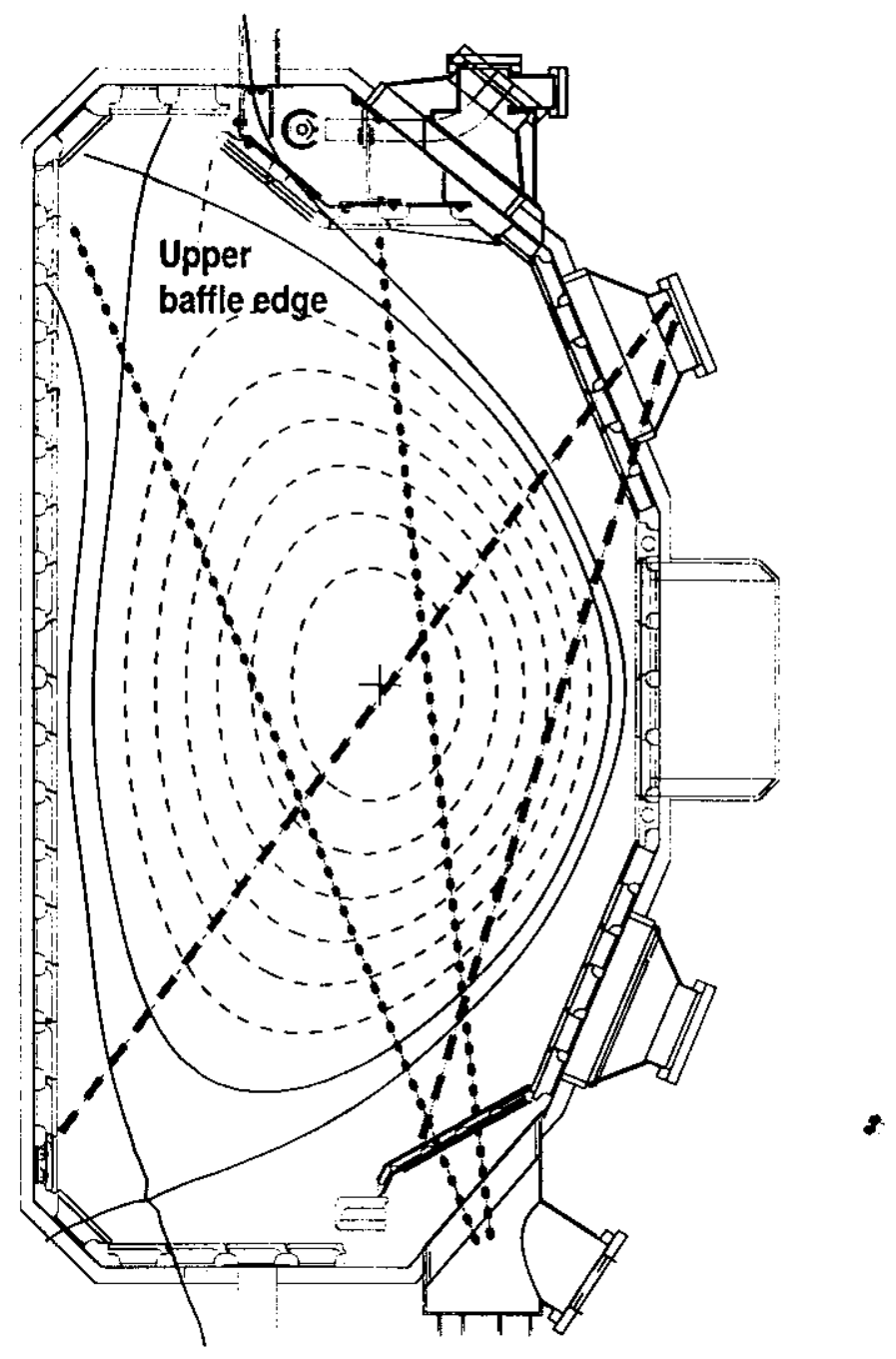

Fig. 1 


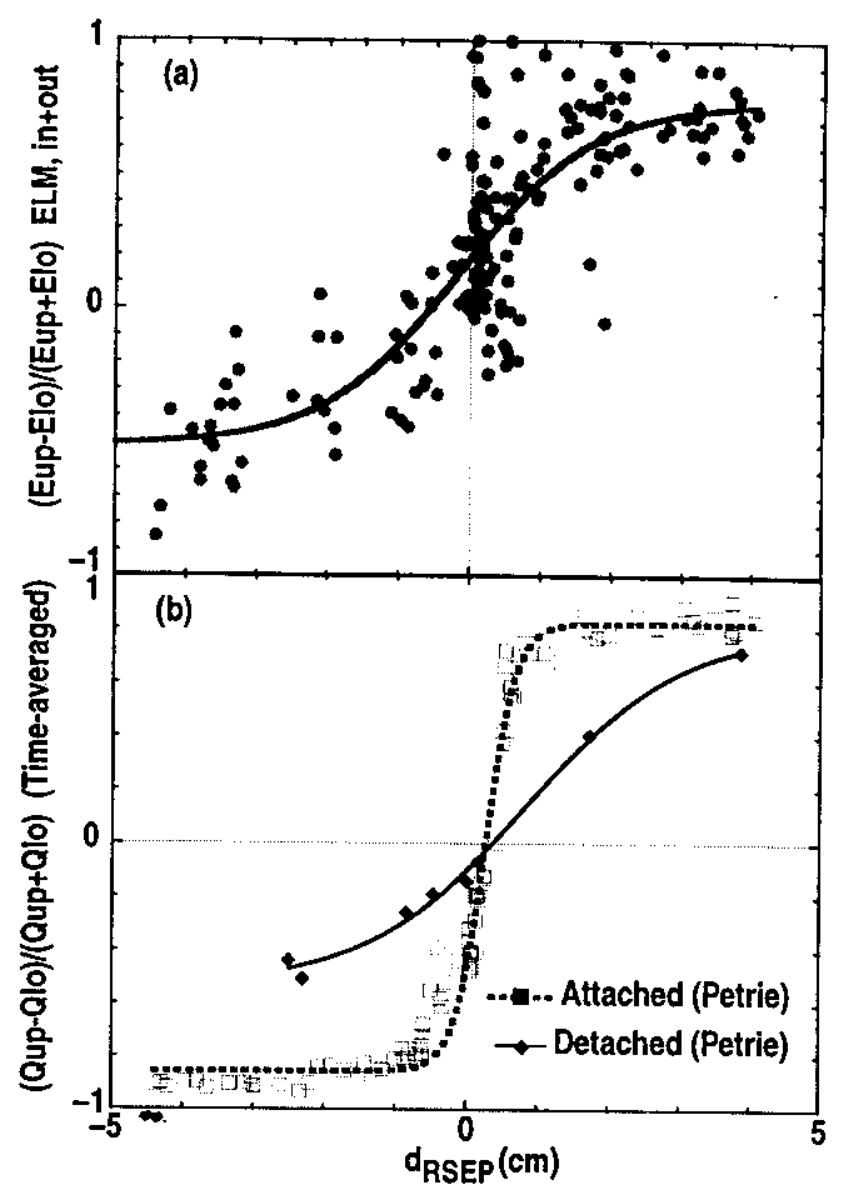

Fig. 2 


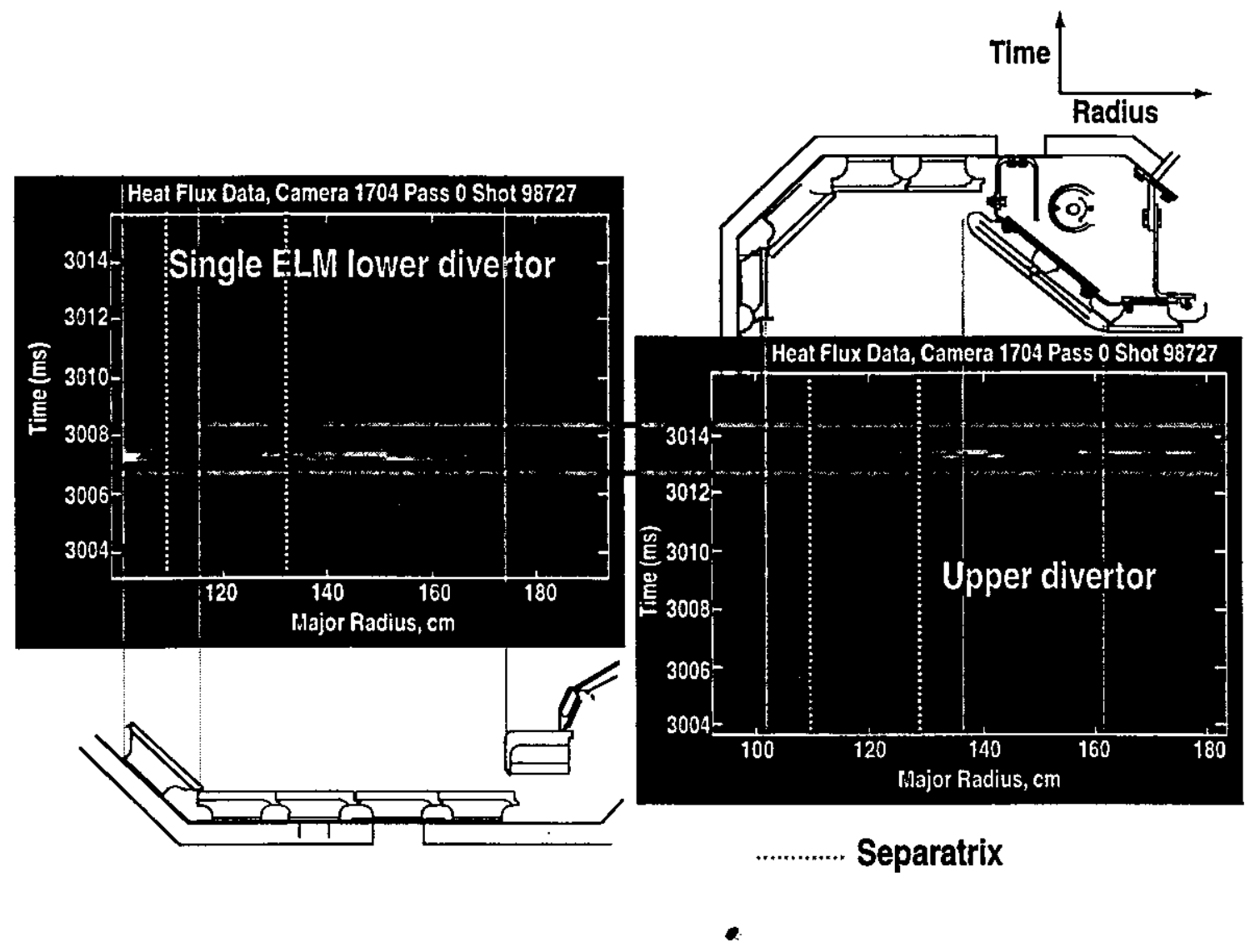



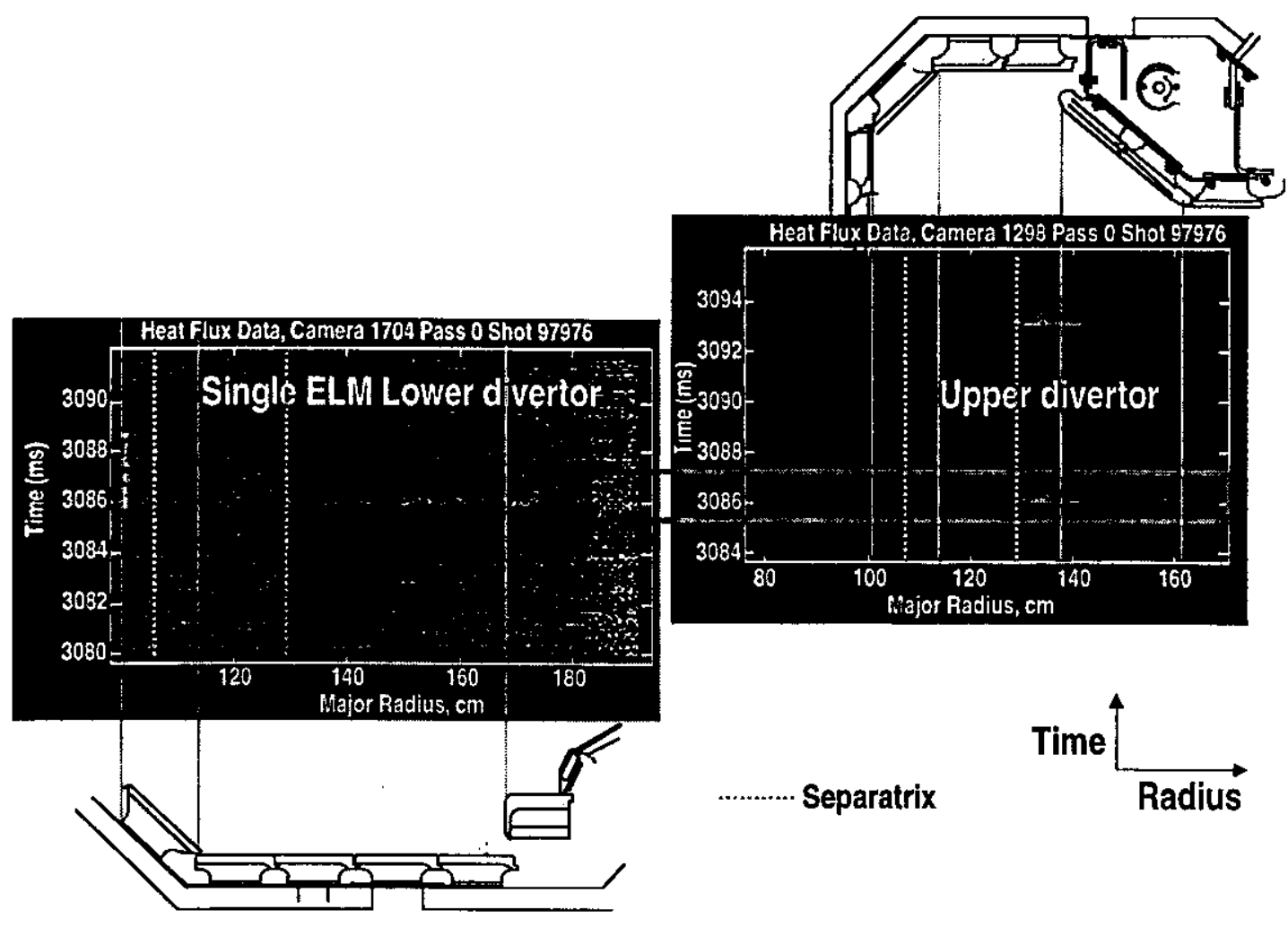

Fig. 4 


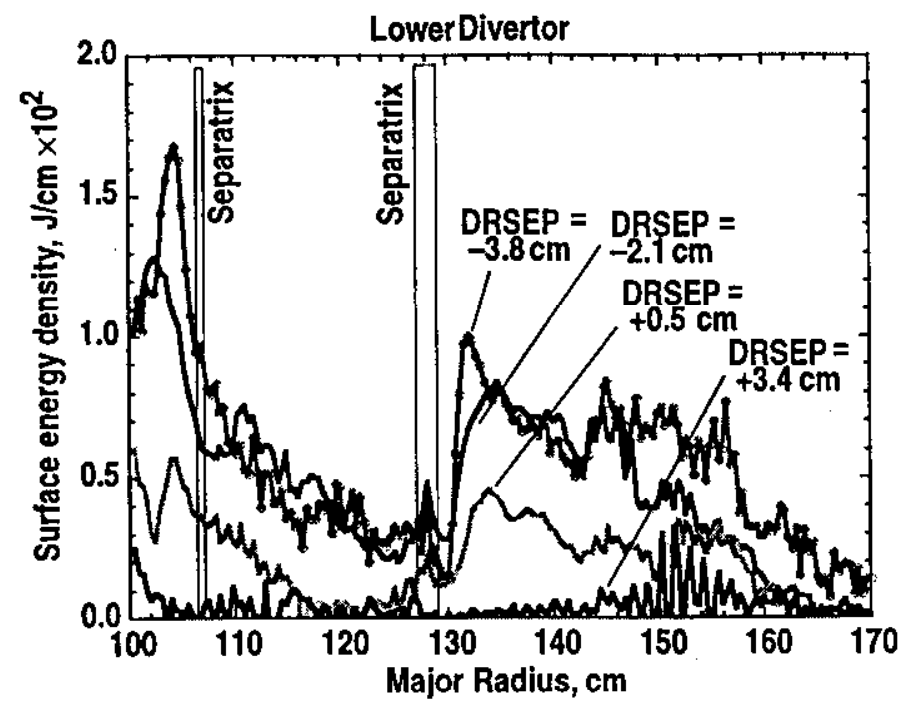




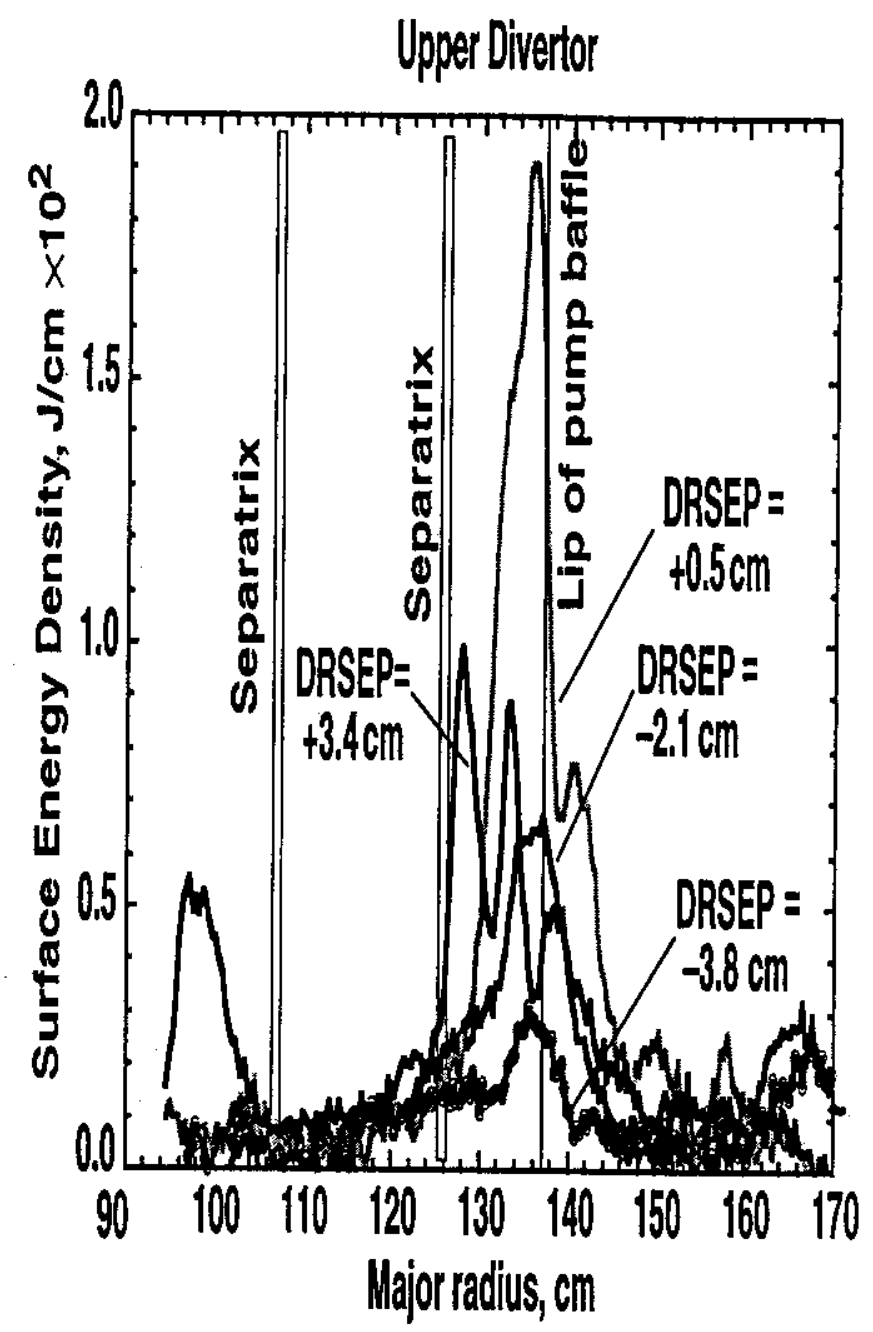

Fig. 6 